\title{
Big-Data, IoT Wearable and mHealth Cloud Platform Integration Triads - a Logical Way to Patient-Health Monitoring
}

\author{
Samyadip Chakraborty, Vaidik Bhatt, Tulika Chakravorty
}

\begin{abstract}
IoT along with big data capabilities is useful in fall detection, medical fridges, sportsman care, patient surveillance, chronic disease management, sleep control and monitoring, etc. Every year a large number of patients are identified with diabetes or cardiac disorders. There is a greater need to handle many patients with the existing medical staff and doctors like cardiologists and diabetologists. This chapter aims at establishing the logical conceptualization and linkages of IoT enabled system linkages with wearable, big-data platforms and cloud-based mhealth delivery. The study subsequently aims at qualitatively and quantitatively validating and putting forth a feasible nuanced understanding framework linking the major contemporary technology triads/automated care delivery process platform in the healthcare context with prime focus on patient health monitoring and care-delivery.

Keywords: detection, medical fridges, sportsman care, patient surveillance, chronic disease management, sleeps control, monitoring.
\end{abstract}

\section{INTRODUCTION}

In the era of Industry 4.0, every industry is using 'Internet of Things' (IoT) for managing and controlling routine operations. The primary purpose behind IoT is empowering objects for connection at any time, anywhere with anything. With these properties of IoT, it is possible to provide new service offering with improvement in the process of existing services. As a sustainable development area in every country, the healthcare sector is also not left behind to use IoT. IoT enhances business intelligence at a hospital and serves the patient in the best way by making patient's vital data available for the physician as well as patients. With the sensors, IoT transmits vital patient-care signs and data of a patient to a remote server, where data is stored, analyzed, and essential patterned information is retrieved from the data. It is essential to monitor ongoing activities and analyze real-time data for providing preventive healthcare services. Health data generated from the sensors of IoTwearables and networked devices are generally voluminous, showcasing variety and high velocity. These large volume, heterogeneous and complex raw data cannot be managed or analysed with traditional data management tools, especially traditional software and hardware platforms;

Revised Manuscript Received on February 05, 2020.

* Correspondence Author

Samyadip Chakraborty, Associate Professor, Department of Operations \& IT, ICFAI Business School (IBS), Hyderabad, India.

Vaidik Bhatt, Research Scholar. Department of Operations \& IT, ICFAI Business School (IBS), Hyderabad, India.

Tulika Chakravorty, Doctoral Scholar, Department of Logistics \& Supply Chain (LSCM), University of Petroleum and Energy Studies, UPES Dehradun, India.

(c) The Authors. Published by Blue Eyes Intelligence Engineering and Sciences Publication (BEIESP). This is an open access article under the CC BY-NC-ND license (http://creativecommons.org/licenses/by-nc-nd/4.0/)

With capability to analyse heterogeneous, autonomous, complex and evolving data, Big-data platforms and its ancillary network process structures finds suitability and effective alignment. With the increase in number of patients afflicted by lifestyle disorders and non-communicable diseases increasing across the globe exponentially; monitoring, analysis and controlling of patients through preventive remedial measures using technology enabled platforms on the move, supported by mobile and cloud platform enabled services has become critical. The storage of such large volume data on physical platforms possess operational challenges in terms of storage, access, linkages and retrieval. Cloud-based service platforms offer important peep through as an apt solution towards this operational issue and offers good scope of providing ubiquitous access and connectivity towards personalized care service proposition and its associated service delivery on the go.

IoT, along with big data capabilities, is useful in fall detection, medical fridges, sportsman care, patient surveillance, chronic disease management, sleep control and monitoring, etc. (Alansari, 2018). On the other hand, digital wearable technologies are also useful for exercise monitoring, heart rate monitoring, female health monitoring, body temperature, blood pressure, sleep cycle monitoring and control, calorie burnt (Lee and Ouyang, 2014) as well as monitoring of blood sugar level (Deshkar et al., 2017; Chen et al., 2018). This chapter tries to highlight and understand the managerial problem linked to assurance of personalized care service delivery and aims at explaining and establishing the logical linkages between how big-data capabilities and IoT enabled cloud-platform helps in achieving superior patient care monitoring. The study puts forth the overarching goal to not only provide a nuanced understanding of how the technology enabled platforms can be synced to offer a superior care-service monitoring regime for the patients, but also extends the propositions and through stepwise scale development technique involving a detailed literature review and expert-driven Q-sort analysis, goes forward towards proposing an operational suggestion of scale-items for the research body of knowledge for understanding and capturing the level of progress for the healthcare setup; no matter how nascent or how lower extent of IoT adoption or Big-data platform implementation.

\section{MANAGERIAL BUSINESS PROBLEM}

India is becoming a hub for lifestyle disorders. Every year many patients are identified with diabetes or cardiac complications. There is a greater need to handle many patients with the existing medical staff and doctors like cardiologists and diabetologists. 
Existing growth rate of healthcare sector in the coming decades will definitely fall short in fulfilling the healthcare needs of an ever growing set of aware patients under epidemiological pressure especially in the backdrop of poor healthcare infrastructure (service divide between rural and urban areas in terms of types of equipment, facilities and healthcare workforce) and limited healthcare workforce. The constant search and quest for advanced and effective preventive care and forecasting ability has been haunting the healthcare community and its stakeholding associates ever since the advent and introduction of technology processes in the healthcare service delivery field. The major challenge for the sector lies in syncing the technology processes and platforms to enhance and put in place suitable analytics driven forecasting ability and data-crunching process platform; enabling synchronous patient health monitoring, data-driven anomaly detection, disease forecasting and most importantly remedial prescriptioning. This problem statement and logical understanding finds more pertinent utility, especially in the context of mobile cloud platform enabled practices which adds to the ability of the care service providers (physicians, nurses, hospitals) to stay a step ahead of the outcome and predict and prevent on the go.

\section{RESEARCH QUESTION}

In this backdrop the major questions and topics of research that surface are:

How IoT driven wearables are helpful for obtaining data for big data analytics?

How Big data implementation and IoT driven wearables impacts cloud-based mHealth?

How cloud-based mHealth enhances the level of patient monitoring?

The backdrop of the business problem embezzled with the relevantly linked three research questions leads towards formulation of the key objectives of this research probing. The three key research questions translates into three consequent and more impactful budding of three research objectives which forms the skeletal framework of this chapter.

The translated Research Objectives give direction to this present study. The key objectives of this study are:

To study the impact of IoT driven wearables on patient health monitoring.

To understand the impact of big data implementation and IoT wearables on cloud-based mHealth for analysing patients' data.

To understand the impact of cloud-based mHealth implementation on patients' health monitoring

\section{LITERATURE REVIEW}

The study systematically highlights and elaborates the extant literature evidences and practitioner literature on Big-data platform implementation, mHealth outcomes IoT-wearables in the context of healthcare sector with predominant emphasis towards patient outcome driven solution implementations.

\section{Big data platform implementation}

With the big data platform implementation, it is possible to study heterogeneous, autonomous, continuous and evolving data (HACE). Data generated from the sensors will be big data and will follow HACE. On the other side, big data can handle large volume, variety, the velocity of data and veracity can be obtained. Big data platform implementation is an extent to which healthcare providers are using big data for warehousing and analysing patients' data.

Big data analytics in the healthcare context is a promising field for providing insights and information from a large dataset. On the other hand, it is helpful in cost reduction also. It is useful in areas of public health, evidence-based medicine, remote monitoring and patient profile analytics. For optimal usage in the healthcare industry, it must overcome the challenges like availability, continuity, ease of use, scalability, ability to manipulate at different levels of granularity, privacy and security enablement, and quality assurance (Raghupathi and Raghupathi, 2014).

Dimiter (2016) gave an operational architecture for implementing IoT based big data analytics in the context of healthcare, pharma and medical devices. Sensor-based IoT devices are an enabler of other medical services like telehealth and telemedicine. With m-IoT and big data implementation, a new category in the healthcare sector will emerge, with the skills and ability to interpret and understand health and wellbeing data which will help patients in their overall health improvement.

\section{Sensor-driven IoT wearables}

Wearable IoT devices have sensors installed in it, and with the sensors, real-time data of vital signs are generated. This generated data help predict the nature of the disease and decides the quality of treatment also. Evidence-based medicines work great with IoT and big data, where medication is prescribed based on the effect of the previous drug on the body. These effects can be analysed using big data analytics generated by the sensor.

Using wearable IoT devices in healthcare improves the quality of life and economic prosperity to medical expert as well as patients. As with the enhanced personal care sensorbased instruments, the patient can always be in contact with the physician. Simultaneously physician can also handle more patients, and scalability for a physician can be generated, which helps in increased quality of care and reducing the medical cost for patient and physician (Alansari et al., 2018).

\section{Cloud-based mHealth}

Could based mHealth platforms provide resources for data storage and data analytics for a large number of patients. With the login credentials, the patient can observe their health-related data on smart-phones and personal computers. A physician can have access to data of all the patients who are under consultation. With the chat window, the patient can always be in the contact of a physician. mHealth apps provide a platform for interaction between patient and physician in a routine as well as a critical situation.

\section{Patient health monitoring}

With sensors where, all vital data can be captured, and big data analytics where all captured data can be analysed, together can help inpatient health monitoring. With the help of various supervised and unsupervised learning and evolving algorithms, it is possible to find a deficiency in health. With predictive algorithms, a physician can take care of a patient with preventive healthcare measures. 
Big data platform, Sensor has driven IoT wearable and Cloud-based mHealth together complete the automatedsystem triad of the big-data IoT interface platform, which is very important for planning and organising workflows and activities enabling data storage.

The data linked to IoT devices and back end Big-data platforms are correctly stored in data stores, which will help in the retrieval of information and in enforcing security. Healthcare analytics demand immediate retrieval of information. The main objective of the triadic Big-IoT automated systems remains to provide for quick and efficient recovery of information. For example, in the electronic medical record (EMR). The doctors can access the data from their office computer or handheld mobile devices on the go even while remotely positioned from patients. So, whenever the patient comes, they can always be ready, and the readiness of care delivery becomes near real-time. The second objective of the automated triads is 'immediate storage of information'. In a manual system, lots of problems are encountered in trying to store a large amount of information.

Big Data Technique-enabled triadic systems extend the benefits to both care-receivers and care-givers through the mediation of integrated systems and big database; thereby saving in many ways, such as Infrastructure-linked aspects and focused cloud readiness. The elements of big-data advantages are Low Cost of Entry \& Scalability, Open Source, Commodity Hardware, UCI Hadoop Ecosystem, Yahoo Hadoop Ecosystem, etc.

\section{Construct definition based on Literature review}

\section{Sensor Driven IoT wearables Adoption}

- The extent to which hospitals use sensor driven IoT wearables for ensuring real-time connectivity, increasing mobility, connecting patient diagnostics/reporting and patient monitoring on the go.

\section{Big-data Platform Implementation}

- The extent to which hospitals use Big-data platform for storage of high velocity large volume medical records, converting past medical records into electronic records, automation of business processes and real time information sharing and analysis.

\section{Cloud-based mhealth practices}

- The extent to which our hospital uses cloud based platform for storage and retrieval of information generated through analytics, keeping all related data together avoiding data redundancy and connecting and sharing important data with stakeholders.

\section{Patient Health Monitoring}

- The extent to which physicians of our hospital can monitor vital patient symptoms, adjust dosage and drug delivery, predicts physiological process-oriented anomalies for patients and suggest remedial measures to patients seamlessly through remote interaction.

\section{Supportive Theoretical base supporting the study Conceptualization}

\section{Temporal Displacement of Care}

Institutions can make value in process by acquiring and examining continuous information of the procedure. Like other industry IT alongside the analytics makes an incentive for human services industry. With presentation of innovation in medicinal services (for example wearable devices and big-data) continuous information of patients can be gotten which can be examined. This replaces the interaction time where specialists and patients connect and related deferrals in giving medicinal services can be controlled which alluded as "Temporal Displacement of Care". With the assistance of temporal displacement, better medicinal services results can be acquired with diminished cost (Thompson et al., 2019). An organization can make an incentive from the dissecting information progressively as action makes place (Lee and Tang, 1997). Care service providers treating lifestyle sickness like diabetes, Chronic Kidney Diseases, Hypertension and other related heart issues can benefit and find parallel from this hypothetical point of view.

\section{Research Gaps}

Based on the extant literature evidences and linkages, certain focus areas of research interest and utility emerge which commands attention and offers potential scope for substantial contribution to the basic body of knowledge linking IoT-wearables, Big-data Platforms and mHealth. Using of IoT devices for customer entertainment and exercise tracking has become quite common these days. However, the implication of the same and the predicted forecasting and remedial prescriptions remains largely unaddressed and limited in healthcare context, and areovertly considered as uncharted area of research. On the other end, despite enhanced and prominent smartphone penetration world-wide, and though mHealth apps and platforms flood mobile-tech domain largely, linkages on the utility and preventive care delivery front in terms of patient care monitoring remains an important new area of development.

Wearable technologies, IoT devices and big data analytics have been studied in details in technical terms, however in the solution oriented specific application enabled linkages and their operationalizations in the realtime sectoral contexts remains largely wanted, let alone in the healthcare sector. Logically and with literature support technological basis behind the technology platforms have been thoroughly established and thus it can be proclaimed that the platforms in isolation or in sync are competent and capable to serve in simulation contexts, however their actual outcome driven solution offering aspect in healthcare domain offers large scope of conceptual and qualitative proclamation and subsequently converting the propositions into testable hypothetical linkages to be established through large-scale empirical studies on the adoption and perceived usefulness of the technology processes and platforms in isolation and in sync in the real business context.

For personalised healthcare service delivery, liberal studies and case-driven empirical explorations exist, however studies linking IoT technology and Big-Data enabled mHealth adoption remains largely lacking. There are very few studies related to lifestyle disorders and that too involving technical aspects or monitoring patient health in the care-delivery context. 
To express in a nutshell a proper measurement tool or a survey based apt item-scale i.e. perceptual measure instrument remains the need of the day to create the fundamental basis and thus establish the data-driven empirical study which will offer the first of its series of studies and thus may offer the stepping stones towards next higher order integration studies for enhanced linkage understandings. There is a need to develop the managerial character for the adoption of technology for managing lifestyle disorders.

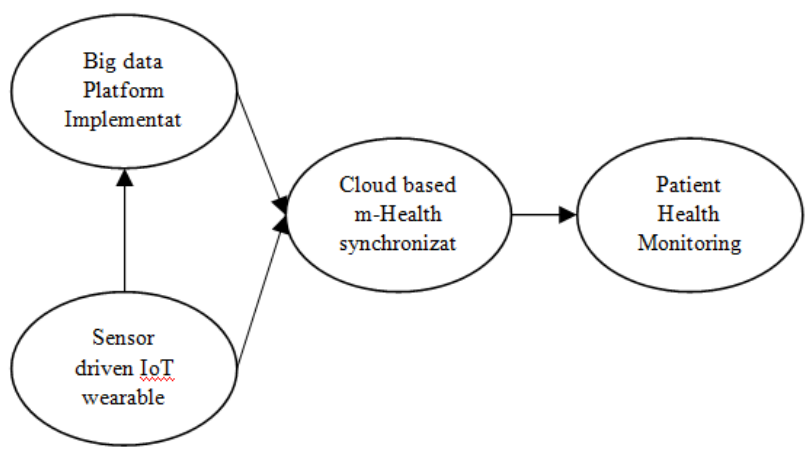

Figure 1 - Proposed Research Model

\section{METHODOLOGY}

The aim of this research lies in not just logical juxtapositioning the relevant concepts but towards theory building, for validating and conforming the theoretical underpinning in a logical sequence through valid steps towards development of the concept and subsequent development of the usable scales to capture and understand the perception of the care service providers.

A systematic and extensive literature review was performed at the first stage which helped in the generation of a pool of items. Second stage, based on a thorough literature review, a relationship model is carefully developed and with the understanding of the theoretical underpinning a working model is proposed. The initial generated pool of items has been ordered and carefully weighted by four academicians as a pre-pilot exercise. In the third stage pilot study is carried out, for calculating the convergent and discriminant validity of the instrument, Q-sort method has been applied (Stephenson, 1953). In the Q-sort method, three sets of academicians and industry professionals having ample research experience in the area of healthcare and technology were invited with industry experts having experience as patient success manager. Q-sort was done for three rounds with the three distinct pairs of experts till the three key metrics of inter-rater reliability, raw agreement score and

Kohen's Kappa values for each round reached above 0.9. this was carried out inorder to ensure that the selected and modified items were suitable for capturing the true essence of the contract objectives.

The experts were having the job roles and profiles of patient success manager and physician success managers were who had the sole objectives of enriching the experience and engagement for patient and physicians.

After providing a description of all constructs and items in brief, the moderator allowed academicians and practitioners to include the item into the category of one or the other category including one group called N/A called not applicable, for omitted items.

Based upon the values of Cohen's kappa (Koch, 1960) and hit ratio (Moore and Benbasat, 1991) results were evaluated. Inter judge agreement score was also checked to measure inter judge reliability and construct reliability. After three rounds of sorting the items, hit ratio, raw agreement score, and Cohen's kappa was found to be above 0.85 . So, after three rounds, the process stopped as inter judge reliability more than 0.8 indicates the perfect agreement between judges and reliable measurement can be obtained.

\section{RESULTS AND ANALYSIS}

With literature review proposed items were selected and inter nudge reliability and validity was checked. After performing three rounds hit ratio, raw agreement score and Cohen's kappa were found to be above 0.90. As the values greater than 0.90 shows perfect agreement between judges, the measurement can be reliable one. After the final round, 4 items for each of the constructs were retained, which claims that, measurement scales are accurate and reliable to measure the underlined abstract phenomenon.

Table 1- Result of Scale development

\begin{tabular}{|c|c|c|c|}
\hline Indices & $\begin{array}{c}\text { Round } \\
1\end{array}$ & $\begin{array}{c}\text { Round } \\
2\end{array}$ & $\begin{array}{c}\text { Round } \\
3\end{array}$ \\
\hline $\begin{array}{c}\text { Hit Ratio } \\
\text { (Placement) }\end{array}$ & 0.77 & 0.84 & 0.94 \\
\hline $\begin{array}{c}\text { Raw } \\
\text { Agreement } \\
\text { Score }\end{array}$ & 0.78 & 0.83 & 0.93 \\
\hline $\begin{array}{c}\text { Cohen's } \\
\text { Kappa }\end{array}$ & 0.66 & 0.77 & 0.91 \\
\hline
\end{tabular}

\section{Measurement Scales}

\section{Sensor Driven IoT wearables Adoption}

Our hospital has been using sensor driven IoT wearables:

- $\quad$ For ensuring real-time connectivity

- For increasing mobility

- For connecting patient diagnostics/reporting

- For patient monitoring on the go.

\section{Big-data Platform Implementation}

Our hospital using Big-data platform

- $\quad$ For storage of high velocity large volume medical records of patient

- $\quad$ For converting past medical records into electronic records

- For automation of business process

- For real time information sharing and analyzing

\section{Cloud-based mhealth practices}

Our hospital is using cloud

- For storage and retrieval of information generated through analytics

- For keeping all related data together

- For avoiding data redundancy

- For connecting and sharing important data with stakeholders 


\section{Patient Health Monitoring}

The physicians of our hospital:

- Monitor vital patient symptoms remotely

- Adjust dosage and drug delivery seamlessly through online interactions

- Warns/forecasts/predicts physical/physiological process-oriented anomalies for patients

- Suggest remedial measures to patients based on symptoms analyzed through IoT wearable datasignals

\section{Proposition Development}

\section{Linking Sensor Driven IoT wearables to Big Data Platform Implementation}

IoT sensor-generated big data cannot be handled solely by cloud-based architecture despite its benefits, as data needs to be processed by efficient computing platforms and scalable algorithms are required to process massive data and to identify patterns from data. With the help of Hadoop distributed file systems (HDFS) and MapReduce based operations data can be easily understood and analysed with pattern finding (Manogaran et al., 2017)

Sensors on the wearable IoT device generates a massive amount of health-related physiological data (Vuppalapati et al., 2016). There is a challenge in integration and real-time presentation of these health-related physiological data, as velocity and volume are high, and not every platform can handle. However, due to its characteristics and capability, big data can not only manage but analyse these massive data. Big data analyses physiological data of a patient in real-time and enables scope for supervised learning for cardiac disorders (Dai et al., 2015). Patient data are extensive in volume with high velocity as sensor senses physiological measures every second and sends data to big data platform. Data provided by sensors of wearables IoT devices are massive and follows the HACE theorem. With its capability to handle heterogeneous, autonomous, continuous and evolving (HACE) data, big data plays a crucial role in handling and analysing health-related data.

\section{Proposition 1: Level of sensor-driven IoT wearables adoption enhances level of big data platform implementation.}

\section{Linking Sensor Driven IoT wearables to cloud-based mHealth}

Cloud architecture provides a holistic approach for EHR as well as sensor system. Cloud-based architecture is essential for enhancing scalability for storage and warehousing of data obtained from IoT sensors (Vuppalapati et al., 2016). Cloud provides essential resources for deploying interfaces which enables communication with the sensors (Doukas and Maglogiannis, 2012). With enabling communication with autonomy between sensors, cloud-based mHealth platforms also provide space for storage of data and facilitate information retrieval. With login credentials, patients and physician accesses data and information stored on cloudbased mHealth platform. For example, data collected from sensors of wearable IoT devices are directly transmitted to the cloud where it is stored and retrieved by patient and physician when it is needed (Yang et al., 2016).
Proposition 2: Level of sensor-driven IoT wearables adoption enhances the cloud-based mHealth

Linking Big Data Platform implementation to Cloud-based mHealth

Big data platform analyses patient's data in real-time using tools like Scala, Hadoop, Hive, Pig etc. It is crucial to store data to decide treatment based on data analysed from big data platform. With proper storage of data, the doctor can learn about the family history of the patient; also, integration between stakeholders can be achieved. Cloud-based mHealth acts as a repository for cleaned and analysed data (Yang et al., 2016). Use of IoT enabled big data analytics in healthcare helps patient in information seeking including nature of the disease and doctor identification before consultation with doctors generates reassurance and ameliorate patient confusion due to information overload. Patient interest in their own health also increases (Iversion, 2008).

Proposition 3: Level of big-data platform implementation enhances the level of cloud-based mHealth.

\section{Linking Cloud-based mHealth to Patient Health Monitoring}

With the new economic model, cloud computing has changed the face of healthcare IT (Kuo, 2011). Data storage on the cloud-based platform is easily retrieved and accessed by a patient as well as physicians via login credentials for health monitoring. A patient can observe their own health via a cloud platform on mobile or personal computer enhances their private participation in maintaining health (Iversion, 2008).

On the other hand, the doctor can also monitor vital signs of patients through remote monitoring. The physician can mentor patients without physical contact from a remote location (Peruman and Manohar, 2017). This monitoring improves the quality of care and decreases the cost for healthcare services (Bin et al. , 2010). A physician can monitor the daily systolic and diastolic blood pressure with deviations to the previous days and can predict future disorder situation, based on past observations (Lan et al. 2012).

\section{Proposition 4: Level of cloud-based mHealth implementation enhances the level of patient health monitoring.}

\section{Model explained with hypothetical example}

A person purchasing wearable IoT device for entertainment, fitness and exercise tracking and health monitoring. As IoT wearable devices have immense capability to measure important vital signs of body like heart rate, blood pressure, temperature, sleep tracking etc with the sensors. On the other hand, device is also useful for exercise tracking like, swimming, jogging, steps climbing etc.

Wearable IoT devices tracks and collects all vital signs in near real-time. As sensors tracks different heterogeneous data continuously, a large volume and high variety data is sent to the big-data platform as well as to the cloud based mHealth platform.

Big data platform used mix of supervised and unsupervised learning for generation of insights. 
From the observed heart rate and blood pressure report with advanced sensor based technology, ECG (electrocardiogram) of a patient can be generated as well as analyzed. With the big-data platform any deviations from normal heart rate, blood pressure and electrocardiogram can be measured accurately. The significant deviation from the normal is accurately analyzed and patient gets notification on mHealth platform about fall detection in case of emergency.

Sensors of the wearable sensors also captures sleep pattern, sleep cycle and body temperature data. Patient can monitor sleep pattern with accuracy. With help of unsupervised learning algorithms big-data platform analyzes acute disease.

Analyzed data by big-data platform as well as raw data from sensors is stored by mHealth cloud services. Patient as well as physicians can log in by their log-im credentials into mHealth services and analyze own health related data on dashboard. Physician can see the common dashboard for all patient. On the other end, dash-board can be customized as per patient - physician requirements.

Big-data platform gives an indication to cloud based mHealth platform in case of critical condition. The indication is given in case if future threats also. Patient as well as physician both have access to the alerts. These alerts displace the time with care, as interaction time between doctor and patient is displaced. Patients do not have to physically go to the physician, however, the physician is able to provide healthcare services from a remote location which increases patient satisfaction as well as ameliorate patients' confusion due to information overload.

\section{IMPLICATIONS}

The theoretical study has an interesting implications. Although the does not empirically claims about adoption and usage of IoT enabled wearable devices for patient monitoring purpose, however, study gives enough evidence from the theory, that using IoT enabled wearable devices will ameliorate patient confusion and increase patient satisfaction, as patient feels that the doctor is there. With real-time physiological data observation, patient proactively participates in healthcare services and cooperate with the physician.

In case of critical as well as routine situation, patient as well as physician both can get the notification with the help of big data platform and cloud based mHealth, and physician can remotely monitor the patient, physician can attempt more patients with economies of scale, and overall cost for healthcare decreases. Private players along with IT companies should take this initiative to develop some supervised and unsupervised healthcare data algorithms for providing better and cost effective healthcare services.

The major contribution of this study is to build theory with identification of the constructs, by which the theoretical model can work. The study is useful for hospital administrator, Private doctors, policy makers and social entrepreneurs. As study affects large group of society which is seeking for good healthcare services at low cost, the study is useful for government policy makers also, in order to form a policy with subsidized rates for wearable devices and most importantly aimed at logical connection and antecedent consequence sync between technology platforms and patient monitoring outcomes.

\section{VIII.LIMITATIONS AND FUTURE OPPORTUNITIES}

The study developed and puts forth scales and conceptualization based on literature and case based evidence stage. Actual adoption and use of IoT enabled wearable devices, big-data platform implementation and cloud based mHealth implementation for patient health monitoring remains yet to be measured empirically based on large-scale data. However, with theory development and literature review enough evidences are provided for the futuristic healthcare sector. The propositions remain to be converted into workable and testable hypotheses for large scale empirical validation of the proposed theory by using elaborate exploratory factor analysis (EFA), followed by confirmatory factor analysis (CFA) and structural equation modelling (SEM), highlighting the path linkages to establish the relationship between wearable devices, big-data platform implementation and patient health monitoring.

\section{CONCLUSION}

The study describes theoretical understanding between sensor driven IoT wearables, big-data platform implementation, cloud based mHealth and its impact on patient health monitoring. Q sort method was also used to develop the measurement scales for the operationalisation of the constructs. With the help of literature theoretical linkage between IoT wearables, big-data platform, clod based mHealth and its impact on patient health monitoring has been established. The study addresses the theoretical understanding with making a foundation for future empirical study via measurement scale development for construct operationalization.

\section{REFERENCES}

1. Alansari, Z., Soomro, S., Belgaum, M. R., \&Shamshirband, S. (2018) The rise of the Internet of Things (IoT) in big healthcare data: review and open research issues. In Progress in Advanced Computing and Intelligent Engineering (pp. 675-685). Springer, Singapore.

2. Bin, S., Yuan, L., Xiaoyi, W.: Research on data mining models for the internet of things. In: Proceedings of International Conference on Image Analysis and Signal Processing, pp. 127-132 (2010)

3. Chen, M., Yang, J., Zhou, J., Hao, Y., Zhang, J., \&Youn, C. H (2018). 5G-smart diabetes: Toward personalized diabetes diagnosis with healthcare big data clouds. IEEE Communications Magazine, 56(4), 16-23.

4. Dai, W., Brisimi, T. S., Adams, W. G., Mela, T., Saligrama, V., \& Paschalidis, I. C. (2015). Prediction of hospitalization due to heart diseases by supervised learning methods. International journal of medical informatics, 84(3), 189-197.

5. Deshkar, S., Thanseeh, R. A., \& Menon, V. G. (2017). A review on IoT based $\mathrm{m}$-Health systems for diabetes. International Journal of Computer Science and Telecommunications, 8(1), 13-18.

6. Dimitrov, D. V. (2016). Medical internet of things and big data in healthcare. Healthcare informatics research, 22(3), 156-163.

7. Doukas, C., \& Maglogiannis, I. (2012, July). Bringing IoT and cloud computing towards pervasive healthcare. In 2012 Sixth International Conference on Innovative Mobile and Internet Services in Ubiquitous Computing (pp. 922-926). IEEE.

8. Gudwani A., M Palash., Puri A., Vaidya M. (2012). India healthcare: Inspiring Possibilities, Challenging Journey, McKinsey health report. https://www.mckinsey.com/featured-insights/india/india-healthcareinspiring-possibilities-challenging-journey (Access Date: $11^{\text {th }}$ October 2019) 
9. Iverson, S. A., Howard, K. B., \& Penney, B. K. (2008). Impact of internet use on health-related behaviors and the patient-physician relationship: a survey-based study and review. Journal of the American Osteopathic Association, 108(12), 699.

10. Koch, J. (1960). A coefficient of agreement for nominal scales. Educational and psychological measurement, 20(1), 37-46.

11. Kuo, A. M. H. (2011). Opportunities and challenges of cloud computing to improve health care services. Journal of medical Internet research, 13(3).

12. Lan, M., Samy, L., Alshurafa, N., Suh, M. K., Ghasemzadeh, H., Macabasco-O'Connell, A., \& Sarrafzadeh, M. (2012, October) Wanda: An end-to-end remote health monitoring and analytics system for heart failure patients. In Proceedings of the conference on Wireless Health (p. 9). ACM.

13. Lee, B. M., \& Ouyang, J. (2014). Intelligent healthcare service by using collaborations between IoT personal health devices. International Journal of Bio-Science and Bio-Technology, 6(1), 155164.

14. Lee, H. L., and Tang, C. S. 1997. "Modelling the Costs and Benefits of Delayed Product Differentiation," Management Science (43:1), pp. 40-53.

15. Manogaran, G., Lopez, D., Thota, C., Abbas, K. M., Pyne, S., \&Sundarasekar, R. (2017). Big data analytics in healthcare Internet of Things. In Innovative healthcare systems for the 21st century (pp. 263-284). Springer, Cham.

16. Moore, G. C., \& Benbasat, I. (1991). Development of an instrument to measure the perceptions of adopting an information technology innovation. Information systems research, 2(3), 192-222.

17. Perumal, K., \& Manohar, M. (2017). A Survey on Internet of Things: Case Studies, Applications, and Future Directions. In Internet of Things: Novel Advances and Envisioned Applications (pp. 281-297). Springer, Cham.

18. Raghupathi W., Ragupathi V. (2014). Bigdata analytics in healthcare promise and potential. Health information science and systems.

19. Thompson, S., Whitaker, J., Kohli, R., \& Jones, C. (2019). Chronic Disease Management: How IT and Analytics Create Healthcare Value Through the Temporal Displacement of Care. Thompson, S., J. Whitaker, R. Kohli, and C. Jones. "Chronic Disease Management: How IT and Analytics Create Healthcare Value through the Temporal Displacement of Care, " MIS Quarterly, Forthcoming.

20. Vuppalapati, C., Ilapakurti, A., \& Kedari, S. (2016, March). The role of big data in creating sense ehr, an integrated approach to create next generation mobile sensor and wearable data driven electronic health record (ehr). In 2016 IEEE Second International Conference on Big Data Computing Service and Applications (BigDataService) (pp. 293296). IEEE.

21. Yang, Z., Zhou, Q., Lei, L., Zheng, K., \& Xiang, W. (2016). An IoTcloud based wearable ECG monitoring system for smart healthcare. Journal of medical systems, 40(12), 286.

\section{AUTHORS PROFILE}

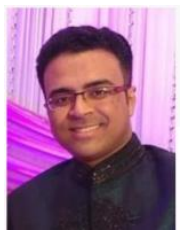

Dr. Samyadip Chakraborty, (Associate Prof and Area Head. Dept of Operations and IT, IBS Hyderabad). He finished his B.Tech from VIT University (Vellore), MBA (UK, University of Liverpool, AACSB Accredited) and $\mathrm{PhD}$ in Operations (IBS Hyderabad). He was a Visiting Fellow researcher to University of Toledo (Ohio, USA). He worked with corporates like TCS, ICICI, NJ India, T. J Hughes (UK), and so forth. He has consulting experience with clients both in India and abroad and has more than 60 research publications in International and national Journals and conferences, including Benchmarking, SCFIJ, Business Theory and Practice, IJAER, IJBI, IJITEE, IJRTE and so on. His areas of teaching and research includes, Information Systems, Business Analytics, Operations Management, Supply chain Management, Healthcare Analytics and Project Management. At IBS Hyderabad, IFHE university, he teaches at various scholastic levels of BBA, MBA, PhD and Executive-MBA.

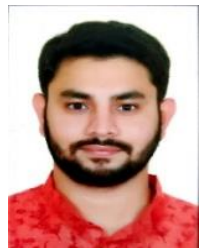

Vaidik Bhatt, (Research Scholar, Dept of Operations and IT, IBS Hyderabad) has finished his B.Pharm from Uka Tarsadia University, Gujarat and MBA from IBS Hyderabad. He is seeking after PhD in Operations from IBS Hyderabad, IFHE University. He has more than two years of involvement with information examination with worldwide customers like Mahindra vehicle, Ford Motors, Nissan, Yamaha India, and so on. His exploration Interest incorporates Healthcare networks, Healthcare Analytics, Digitization of critical care services, Technology consideration in Healthcare and so forth. He has publications in different worldwide meetings and Journals like ISDSI, IJPHRD, IJITEE, IJRTE and so forth.

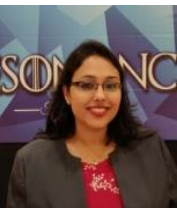

Tulika Chakravorty, (Doctoral Research Scholar of Logistics \& Supply Chain Management, from University of Petroleum and Energy Studies, UPES Dehradun). She has completed her B.Tech from Integral University, Lucknow and M.S in Quality Management from BITS Pilani. She is currently working as senior consultant and corporate trainer with Accenture Solution Pvt Ltd, Hyderabad, having 10 years of corporate experience in various domains like supply chain, Healthcare, Banking, Insurance etc. with various domestic and international clients like AstraZeneca, JP Morgan Chase, Samsung etc. Research area is Healthcare management focusing on Digitization of Hospital supply chain using IoT Technologies. She has got over 20 publications in International conferences and Journals including IJBI, IJPHRD etc. 